\title{
Why Zeolites Have So Few 7-Membered Rings
}

\author{
Xi Li ${ }^{\dagger}$ and Michael W. Deem ${ }^{*, \dagger, \dagger}$ \\ Department of Bioengineering, Rice University, Houston, TX 77005 \\ E-mail: mwdeem@rice.edu
}

${ }^{*}$ To whom correspondence should be addressed

${ }^{\dagger}$ Department of Bioengineering, Rice University, Houston, TX 77005

${ }_{\ddagger}^{\ddagger}$ Department of Physics \& Astronomy, Rice University, Houston, TX 77005 


\begin{abstract}
Known zeolite structures have relatively few 7-membered rings. Previous quantum mechanical calculations suggest there is no particular energy penalty for 7-membered rings. Predicted zeolite structures sampled from all possible symmetries also do not explain why there are so few observed 7-membered rings. Here, we analyze the ring size distributions of predicted structures as a function of energy and density. We show that predicted structures with low density, in the range where known zeolites exist, have relatively few 7-membered rings. It appears that the constraint of proximity to the lowdensity edge of predicted structures is what leads to a low probability of 7 -membered rings. These results suggest that low-density predicted structures are similar to known zeolites and of greatest interest as new synthetic targets.
\end{abstract}

\title{
Keywords
}

zeolites, 7-rings

\section{Introduction}

Zeolites are fascinating examples of $\mathrm{SiO}_{2}$ composition leading to a dramatic diversity of architectures. ${ }^{1}$ There are over 200 known zeolite topologies, most of which can be made synthetically, roughly 50 of which are naturally occurring. ${ }^{2}$ The pore and channel structures of zeolites make these materials selective adsorbants and catalysts. For example, the ring size of the apertures of zeolite frameworks varies between different topologies, and the results of kinetic accessibility and catalytic test reaction are often interpreted in terms of the limiting ring size in a given material. Here, for instance, an "7-ring" refers to a closed loop that is built from seven tetrahedrally coordinated silicon (or aluminum) atoms and seven oxygen atoms. ${ }^{3}$ These rings are not always perfectly flat or symmetrical due to a variety of effects, including strain induced by the bonding with atoms not in the ring or coordination of some 
oxygen atoms of the rings to cations within the structure. ${ }^{4,5}$ Therefore, the pore openings for all rings of one size are not identical. ${ }^{6}$ Nonetheless, ring distribution is a fundamental characteristic of a zeolite structure.

It is remarkable how few known zeolites contain seven rings. Early quantum mechanical studies were carried out to determine whether rings containing seven Si atoms were energetically unfavorable. ${ }^{7}$ These studies conclude there is no energetic bias against 7 -membered rings. Most zeolites are highly symmetrical, and in monoclinic, rhombohedral, tetragonal or trigonal/hexagonal systems there is one unique axis which has higher rotational symmetry than the other two axes and which might inhibit odd-membered rings. Studies that sample the entire space of symmetrical structures, however, also do not show a general bias against 7-membered rings. ${ }^{8,9}$ Other general physiochemical properties of predicted zeolite structures, such as energy, density, X-ray diffraction patterns, ring-size distributions other than seven, and $\mathrm{I} / \mathrm{I}(\mathrm{cor})$ are in good agreement with those from known zeolites. Thus, previous work presents a conundrum of why there are so few 7-membered rings in known zeolites.

Predicted zeolite structures are often parametrized by their energy and density. Indeed, most of the known zeolites lie near a line, the distance from which is termed the feasibility factor. ${ }^{10}$ This line is at the low-density edge of the distribution of predicted structures, presumably because zeolites nucleate from a low-density solution, and a zeolite nucleates as soon as the density is high enough to be within the distribution of possible structures. ${ }^{9}$ Thus, known zeolites lie at the low-density edge of predicted, thermodynamically possible structures.

Not only synthesizability, but also other general features of predicted zeolites may depend on their location in density-energy space. In particular, the probability of a 7-membered ring in a structure may depend on the coordinates in density-energy space. In this work, we measure the ring size distribution as a function of distance from the low-density edge of the distribution of predicted structures. This paper is organized as follows. Section 2 describes the Monte Carlo procedure used to sample the space of predicted zeolite materials and the 
methods for construction of the ring size distributions as a function of density and energy. Section 3 presents the histograms of ring distributions, as a function of distance from the low-density structures. Section 4 discusses the results, focusing on how the probability of 7-membered and 9-membered rings differ between the low-density structures and higherdensity structures. We conclude in section 5 .

\section{Methods}

The database of predicted zeolite structures contains over $2.6 \mathrm{M}$ distinct topologies. ${ }^{9}$ The database was created by a Monte Carlo sampling of a zeolite Hamiltonian, given constraints of symmetry and unit cell size. Unit cell sizes consistent with known zeolites and all space groups were examined in the construction of the database. Si atom positions were determined from the Monte Carlo sampling and then optimized to determine final Si and O atom positions in the unit cell. Two distinct interatomic potentials, Sanders-Leslie-Catlow (SLC) and van Beest-Kramer-van Santen $(\mathrm{BKS})$, were used. ${ }^{11,12}$ For each structure, the atomic positions as well as several structural properties such as unit cell parameters and symmetry, density, computed energy, dielectric constant, compressibility, and ring distributions were computed. There are over $330 \mathrm{k}$ structures within $+30 \mathrm{~kJ} / \mathrm{mol} \mathrm{Si}$ of $\alpha$-quartz in the SLC database and over $590 \mathrm{k}$ within $+65 \mathrm{~kJ} / \mathrm{mol}$ Si of $\alpha$-quartz in the BKS database. We note that BKS energy differences must be scaled by a factor of one-third to agree with experiments. These structures are considered to be thermodynamically accessible and hence especially interesting from the point of view of synthesis of novel zeolites. We here analyzed the database of structures with the SLC interatomic potential, including only structures within $+30 \mathrm{~kJ} / \mathrm{mol} \mathrm{Si}$ of $\alpha$-quartz, as the SLC interatomic potential is generally regarded as accurate for zeolites. ${ }^{13}$

Most known zeolites are near a line in density energy space. ${ }^{10}$ The distribution of predicted structures and this line are shown in Fig. 1. In the present calculations, structures 

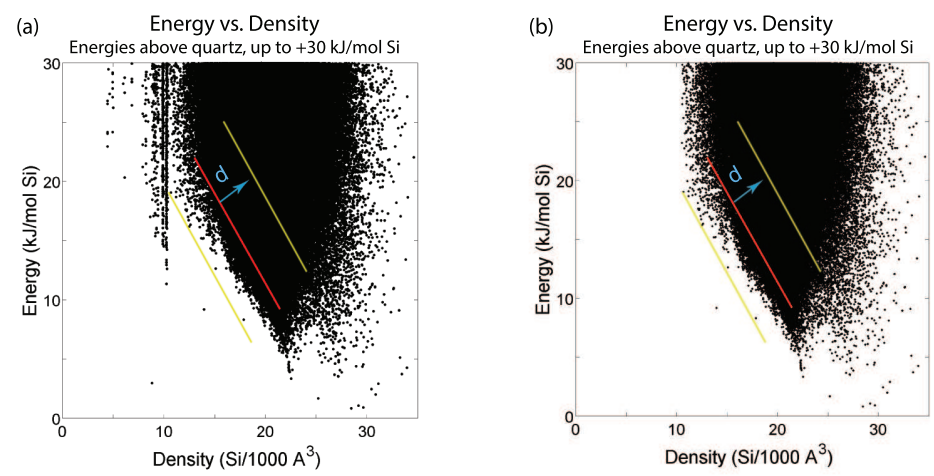

Figure 1: Energy-density distributions for computationally predicted zeolite structures. a) All predicted structures. A linear fit to the known zeolites is shown as a red line. ${ }^{9} \mathrm{~b}$ ) Structures, excluding those with density below $10 \mathrm{Si} / 1000 \AA^{3}$. The signed feasibility factor, ${ }^{10}$ $d$, is the distance from the linear fit to the known zeolites. The signed feasibility factor is the length of the blue arrow, positive to the right of the red line, and negative to the left. Known zeolites have low absolute values of $d$. All predicted structures between the two yellow lines were used in the calculation of the ring histograms.

with ultra-low densities below $10 \mathrm{Si} / 1000 \AA^{3}$ were excluded. Structures were classified by their feasibility factor, ${ }^{10} d$, the signed distance from the line fit to known structures. ${ }^{8}$ The properties of the predicted structures in bands from $d$ to $d+1$ were calculated. The nine intervals in the range from -4 to +6 were considered. Most of the known zeolites lie in this range.

The ring size distributions were calculated for each band $d$ to $d+1$. A ring is defined by the number of Si atoms within it. The size distributions were calculated for fundamental rings, and a fundamental ring was defined as a ring that cannot be divided into a set of smaller rings. ${ }^{5,14}$ The minimum number of bonds between any two atoms in a fundamental ring is given by the number of bonds that separate them on the ring; there is no other shorter path connecting them in the rest of the zeolite. Ring sizes from 3 to 20 were tabulated for each zeolite. Ring histograms were computed per unit cell of the zeolite, and each fundamental ring identified was weighted by its fraction contained within the primary unit cell. Ring histograms from different structures were averaged within the band $d$ to $d+1$, where each structure was weighted by its unit cell volume. Figure 1b shows the distribution of zeolites 
for which ring histograms are calculated.

\section{Results}

\section{Density and energy distribution}

The density-energy distribution of predicted zeolite structures in the SLC database is shown in Fig. 1a. There are two parts to the distribution. There is a main distribution with densities above $10 \mathrm{Si} / 1000 \AA^{3}$. This is the distribution used in the present work, and shown in Fig. 1b. There is also a low-density distribution with densities below $10 \mathrm{Si} / 1000 \AA^{3}$. While several intriguing zeolites with densities in this range have recently been synthesized, ${ }^{15,16}$ this part of the distribution was not considered in the present work. The limits of $d=-4$ and $d=5$ shown in Fig. 1b define the low-density structures that are the focus of the present work.

\section{Ring Histograms Depend on the Feasibility Factor}

Histograms of ring sizes were calculated for feasibility factors between $d=-4$ to 5 , in bands of $d$ to $d+1$. Results are shown in Fig. 2. We see that the probability of a seven ring decreases as the density decreases from the central part of the distribution to the low-density edge of the distribution. That is, the results show known zeolites, which have a low value of $|d|$, should have a smaller probability of a seven ring than does the average zeolite in the predicted database. The known zeolites used to defined the feasibility factor lie in the range $[-3,10] .^{8}$

\section{Probability of 7-Membered Rings Depends on the Feasibility Factor}

One of the most striking results of Fig. 2 is the dependence of the probability of a 7-membered ring on the feasibility factor. In Fig. 3, this probability is displayed as a function of the feasibility factor. This probability is shown for the nine bands of structures considered, 

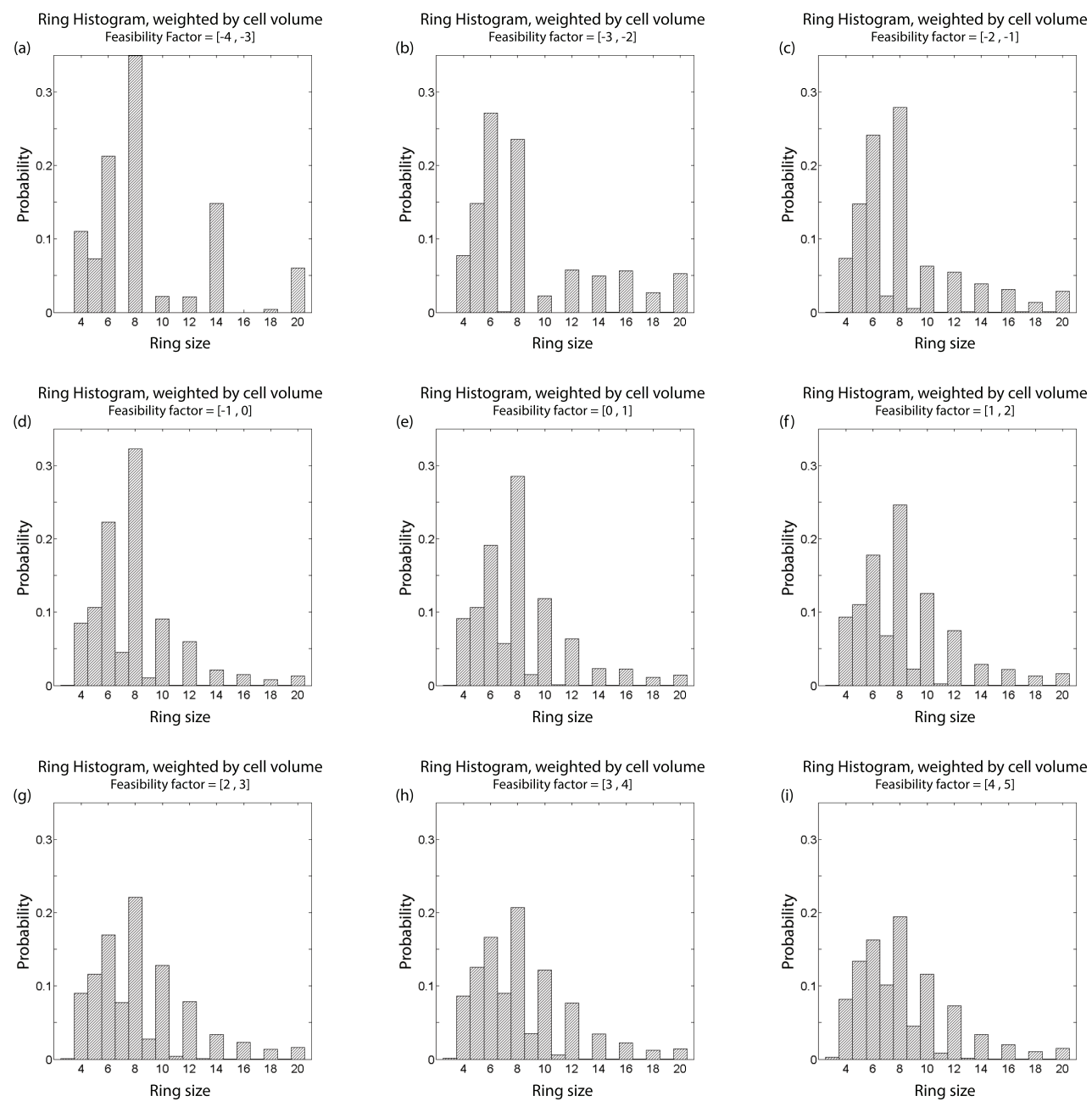

Figure 2: Ring size distributions according to different feasibility values. Results are shown for each stripe $d$ to $d+1$. Note that the probability of a 7-membered ring decreases as $d$ is reduced to the low-density edge of the distribution of predicted structures. 
those within the yellow lines of Fig. 1b.

\section{Probability of 7-Membered Ring}

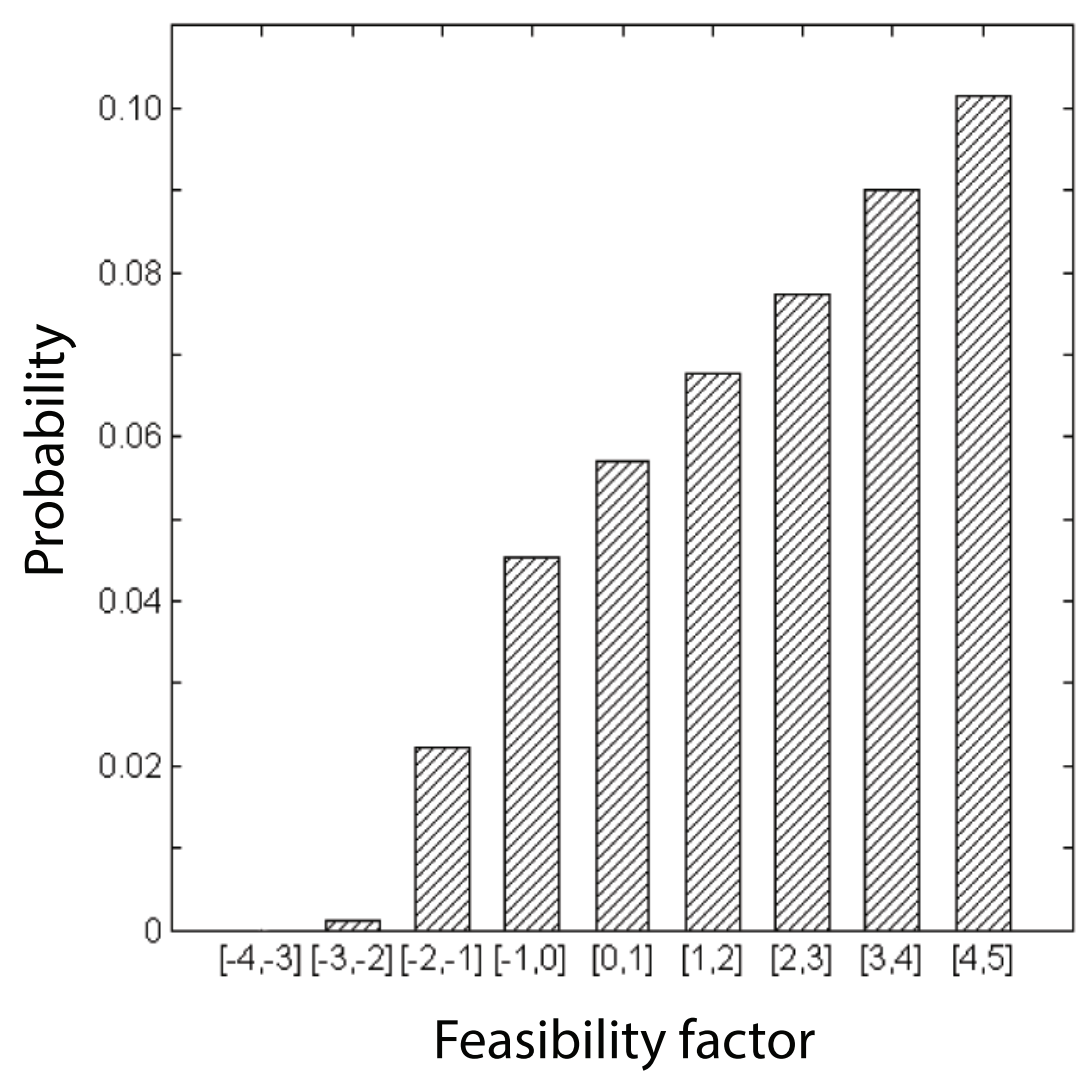

Figure 3: The probability of a 7-membered ring, as it depends on the feasibility factor. These data were taken from Fig. 2.

\section{Discussion}

The database provides an unbiased sample of possible zeolite structures. What we have shown is that known zeolites lie in a range of energy-density space in which 7-rings are rare. This result is not a priori obvious.

Zeolites are often thought to be metastable species, at least in the absence of organic structure directing agents and synthesis solution conditions, perhaps kinetically determined. 
Indeed, kinetic limitations likely explain why zeolites are at the low density edge of predicted structures. As a zeolite nucleates from a low density solution to a condensed phase, the density increases, and when the trajectory in energy-density space reaches the low-density edge of the distribution of possible zeolites, Fig. 1a, a zeolite nucleates. ${ }^{9}$ Thus, most known zeolites are at the low-density edge of the distribution of possible zeolites.

The present results show that the kinetic limitation to be at the low density edge of possible structures implies a reduction in the probability of 7-rings. As shown in Fig. 3, the probability of observing a 7-membered ring in a predicted zeolite increases with increase of the signed feasibility factor. Known zeolites are at lower values of the feasibility factor, near the low-density edge of the distribution of possible zeolites, as shown in Fig. 4. The feasibility factor range of $[-3,1]$ covers nearly 80 percent of all known structures. The histogram of ring sizes is shown for the known zeolites in Fig. 5. Therefore, known zeolites have a lower probability of 7 ring than the bulk of the predicted structures in the SLC database. Alternatively, one may say that the low-density edge of the distribution of predicted structures is most representative of the currently known zeolites. ${ }^{17}$ The best agreement between the predicted structures and the known structures appears in the feasibility factor range of $[-3,-1]$.

As noted above, there is agreement between the known zeolite structures and the predicted structures for the probability of 7 -membered rings in the range [-3,1], in which most known feasibility factors lie. This result explains why there are so few seven rings in the known zeolites: the database of predicted structures shows there are few seven rings in structures with feasibility factors less than 1 . That is, 7-membered rings are relatively rare in Figs. 2a-e compared to the rest of Fig. 2, the average of which is shown as Fig. 2a in Ref. ${ }^{9}$

In the known zeolites, ring size 3 and 9 are correlated and tend to occur simultaneously in the same structure. This trend is demonstrated by a comparison of Fig. 5a and Fig. 5b. While some 3-membered rings occur in the predicted structures, they are highly strained and are not common. In known zeolites, 3-membered rings usually require the presence of 


\section{Feasibility Distribution of Known Zeolites}

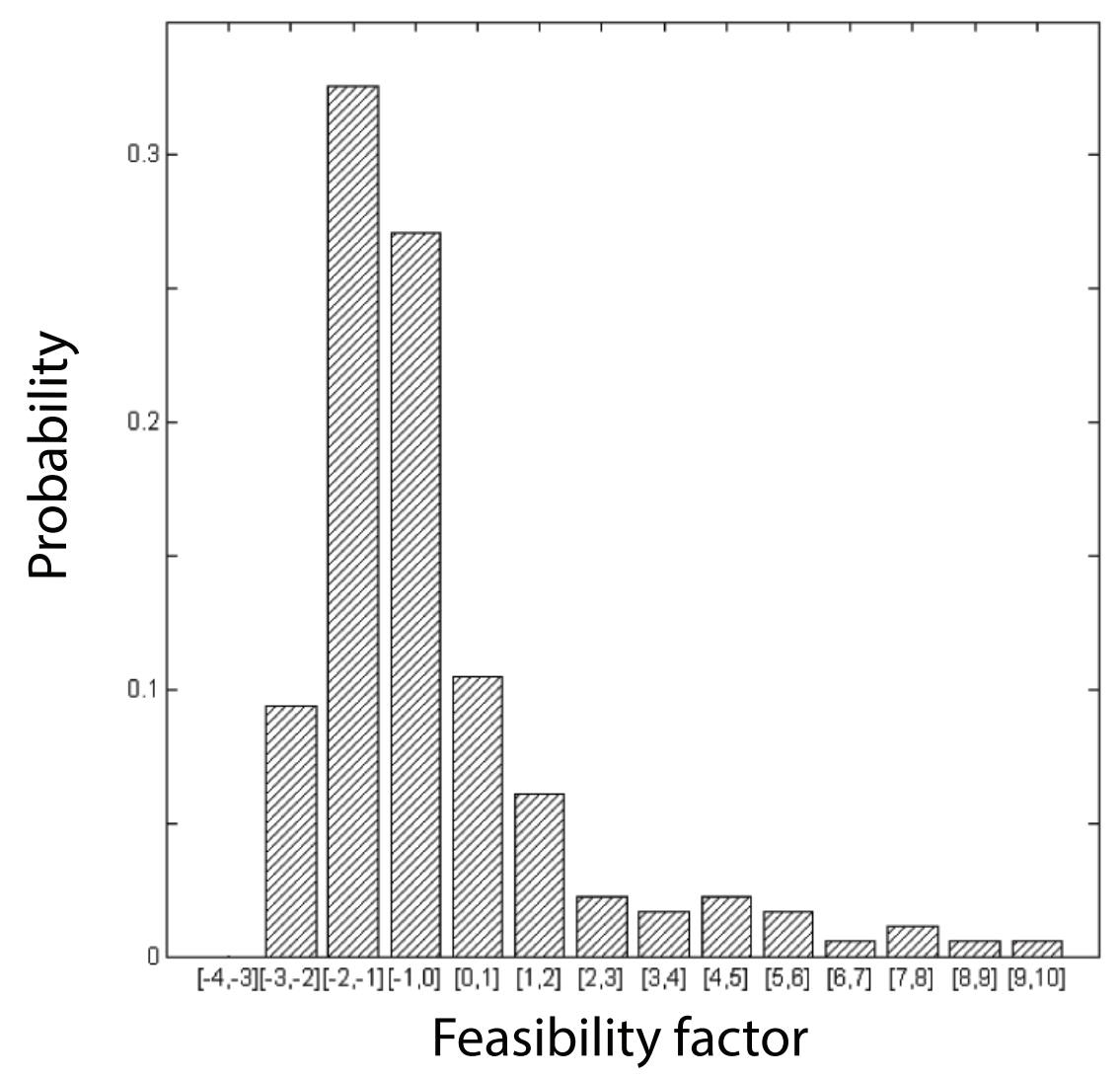

Figure 4: Histogram of the known zeolites as a function of the signed feasibility factor. These structures cluster around the red line in Fig. 1.
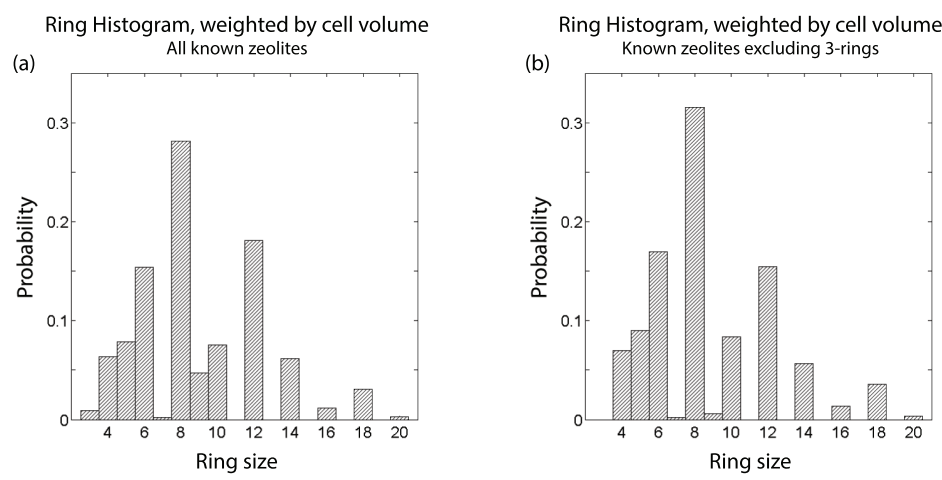

Figure 5: Ring size distribution of the known zeolite structures. a) All known zeolites, b) Known zeolites, excluding structures with 3-membered rings. Note 9-membered rings are rarely observed in the absence of 3 -membered rings. 
heteroatoms such as beryllium, zinc, or germanium. ${ }^{18}$ Thus, there are more 9 rings in the known zeolites than in the predicted structures because the predicted pure silica structures have fewer 3-membered rings than do the known structures. The histograms of known structures in Fig. 5b are similar to the histogram from the low-density predicted structures, with feasibility factor in the range $[-3,-1]$.

Many properties of the bulk distribution of predicted zeolites are similar to that of the known zeolites. Other than the probability of 7 -membered rings, the ring histograms are quite similar. For example, the probability of odd-membered, large rings is low in both the predicted and known zeolite structures. Other properties of the predicted structures such as powder X-ray diffraction patterns are also similar to that of known zeolites. ${ }^{9}$

These results for the dependence of the 7-ring probability on the feasibility factor illustrate the value of partitioning the database of predicted structures into low- and high-density materials. ${ }^{17}$ While some of the properties of the materials may be relatively uniform in the density-energy space, others may be more sensitive to the coordinates in density-energy space. For example, in addition to the probability of 7-rings, the ease of synthesis also depends on the feasibility factor. It may be the case that other interesting features depend on the feasibility factor. Furthermore, it may be the case that known zeolites and the predicted materials may differ in more subtle ways that depend on variables in addition to the feasibility factor. Further screens of the database for performance metrics and, hopefully, synthesis of some of the predicted structures will shed light on the distributions of known, possible, and predicted zeolite structures.

This database may be used to identify newly made materials, search for novel materials with defined function, or motivate the synthesis of new materials. For example the recently discovered ITQ- $40^{16}$ is an example of a 16 -membered ring structure whose general class was predicted to exist a decade ago before any such materials were known. ${ }^{5}$ The recently discovered ITQ- $51^{19}$ is another example of a 16 -membered ring zeolite and is in the database. ${ }^{9}$ In the search for new known materials in the database of predicted structures, low-density 
predicted structures should be preferentially examined. Conversely, when screening the database of predicted structures for potential applications, low-density structures should be given preferential weight, as was done in the screening of carbon capture materials. ${ }^{17}$

\section{Conclusion}

The observed frequency of 7-membered rings in zeolite structures is lower than previous energetic, quantum mechanical, and crystallographic arguments can explain. A partitioning of the database of predicted zeolite structures by density and energy was considered. It was shown that the probability of 7 -membered rings decreases as the density is decreased toward the low-density edge of the distribution of predicted zeolites. Thus, not only does the feasibility of zeolite synthesis increase as density is lowered, but also does the probability of a 7-membered ring decrease.

The database of computationally predicted zeolite-like materials contains over $2.6 \mathrm{M}$ unique structures. Roughly 330k of the materials in the SLC portion of the database lie within the $+30 \mathrm{KJ} / \mathrm{mol}$ Si energetic band above $\alpha$-quartz in which the known zeolites lie. Additionally, $80 \%$ of the known zeolites have a feasibility factor less than 1 . The subset of the database of predicted structures in this feasibility range shows a reduced 7-membered ring probability, consistent with the known zeolites. These low-density predicted structures are of most interest in screening of the database for new functional materials to be synthesized with current zeolite synthesis techniques. Of the 330k predicted structures, 26,282 lie in the low-dimensional feasibility factor range of $[-3,1]$. Perhaps the evolutionary method to design

chemically-synthesizable linkers will aid in the synthesis of some of these new, predicted materials. ${ }^{20,21}$ 


\section{Acknowledgments}

This research was supported by the Office of Basic Sciences of the US Department of Energy under grant DE-FG02-03ER15456.

\section{References}

(1) Blatov, V. A.; Ilyushin, G. D.; Proserpio, D. M. The Zeolite Conundrum: Why Are There so Many Hypothetical Zeolites and so Few Observed? A Possible Answer from the Zeolite-Type Frameworks Perceived As Packings of Tiles. Chem. Mater. 2013, 25, $412-424$.

(2) Baerlocher, C. H.; Meier, W. M.; Olson, D. H. Atlas of Zeolite Framework Types; Elsevier: Amsterdam, 2001; http://www.iza-structure.org/.

(3) Acton, Q. A. Reactive Nitrogen Species - Advances in Research and Application; ScholarlyEditions: Atlanta, 2013; http://books.google.com/.

(4) Dumrul, S.; Bazzana, S.; Warzywoda, J.; Biederman, R. R.; Jr., A. S. Imaging of Crystal Growth-Induced Fine Surface Feartures in Zeolite A by Atomic Force Microscopy. Micropor. Mesopor. Mater. 2002, 54, 79-88.

(5) Curtis, R. A.; Deem, M. W. A Statistical Mechanics Study of Ring Size, Ring Shape, and the Relation to Pores Found in Zeolites. J. Phys. Chem. B 2003, 107, 8612-8620.

(6) Fuoco, D. A New Method for Characteriazation of Natural Zeolites and Organic Nanostructure Using Atomic Force Microscopy. Nanomaterials 2012, 2, 79-91.

(7) Hacarlioglu, P.; Lee, D.; Gibbs, G.; Oyama, S. Activation Energies for Permeation of He and $\mathrm{H}_{2}$ through Silica Membranes: An ab initio Calculation Study. J. Membr. Sci. 2008, 313, 277-283, DOI: 10.1016/J.MEMSCI.2008.01.018. 
(8) Deem, M. W.; Pophale, R.; Cheeseman, P. A.; Earl, D. J. Computational Discovery of New Zeolite-Like Materials. J. Phys. Chem. C. 2009, 113, 21353-21360.

(9) Pophale, R.; Cheeseman, P. A.; Deem, M. W. A Database of New Zeolite-Like Materials. Phys. Chem. Chem. Phys. 2011, 13, 12407-12412, DOI: 10.1039/C0CP02255A.

(10) Dorota Majda, F. A. A. P.; Friedrichs, O. D.; Foster, M. D.; Simperler, A.; Bell, R. G.; Klinowski, J. Hypothetical Zeolitic Frameworks: In Search of Potential Heterogeneous Catalysts. J. Phys. Chem. C 2008, 112, 1040-1047.

(11) Schroder, K.-P.; Sauer, J.; Leslie, M.; Catlow, C. R. A.; Thomas, J. M. Bridging Hydrodyl Groups in Zeolitic Catalysts: A Computer Simulation of their Structure, Vibrational Properties and Acidity in Protonated Faujasites ( $\mathrm{H}-\mathrm{Y}$ Zeolites). Chem. Phys. Lett. 1992, 188, 320-325.

(12) van Beest, B. W. H.; Kramer, G. J.; van Santen, R. A. Force fields for silicas and aluminophosphates based on ab initio calculations. Phys. Rev. Lett. 1990, 64, 19551958.

(13) Zwijnenburg, M. A.; Cor, F.; Bell, R. G. On the performance of DFT and Interatomic Potentials in Predicting the Energetics of (Three-Membered Ring-Containing) Siliceous Materials. J. Phys. Chem. B 2009, 111, 6156-6160.

(14) Stixrude, L.; Bukowinski, M. S. T. Rings, Topology, and the Density of Tectosilicates. Amer. Mineral. 90, 75, 1159.

(15) Sun, J. L.; Boeau, C.; Catin, A.; Corma, A.; Diaz-Caban̄as, M. J.; Moliner, M.; Zang, D. L.; Li, M. R.; Zou, X. D. The ITQ-37 Mesoporous Chiral Zeolite. N 2009, $458,1154-1159$.

(16) Corma, A.; Diaz-Caban̄as, M. J.; Jiang, J.; Afeworki, M.; Dorset, D. L.; Soled, S. L.; Strohmier, K. G. Extra-large pore zeolite (ITQ-40) with the Lowest Framework Desity 
Containing Double Four- and Double Three-rings. Proc. Natl. Acad. Sci. USA 2010, 107, 13997-14002.

(17) Lin, L.-C.; Berger, A. H.; Martin, R. L.; Kim, J.; Swisher, J. A.; Jariwala, K.; Rycroft, C. H.; Bhown, A. S.; Deem, M. W.; Haranczyk, M. et al. In Silico Screening of Carbon-Capture Materials. Nature Materials 2012, 11, 633-641, DOI: 10.1038/NMAT3336.

(18) Cejka, J.; Corma, A.; Zones, S. Zeolites and Catalysis: Synthesis, Reactions and Applications; Wiley-VCH: Weinheim, 2010; http://books.google.com/.

(19) Martinez-Franco, R.; Moliner, M.; Yun, Y. F.; Sun, J. L.; Wan, W.; Zou, X. D.; Corma, A. Synthesis of an Extra-Large Molecular Sieve using Proton Sponges as Organic Structure-Directing Agents. Proc. Natl. Acad. Sci. USA 2013, 110, 3749-3754.

(20) Pophale, R.; Daeyaert, F.; Deem, M. W. Computational Prediction of ChemicallySynthesizable Organic Structure Directing Agents for Zeolites. Phys. Chem. Chem. Phys. 2013, 1, 6750-6760.

(21) Davis, J.; Deem, M. W.; Davis, M. E. Synthesis of a Specified, Silica Molecular Sieve Using Computationally Predicted Organic Structure Directing Agents. Agnew. Chem. Int. Ed. 2014, DOI: 10.1002/anie.201404076R1. 


\section{Graphical TOC Entry}

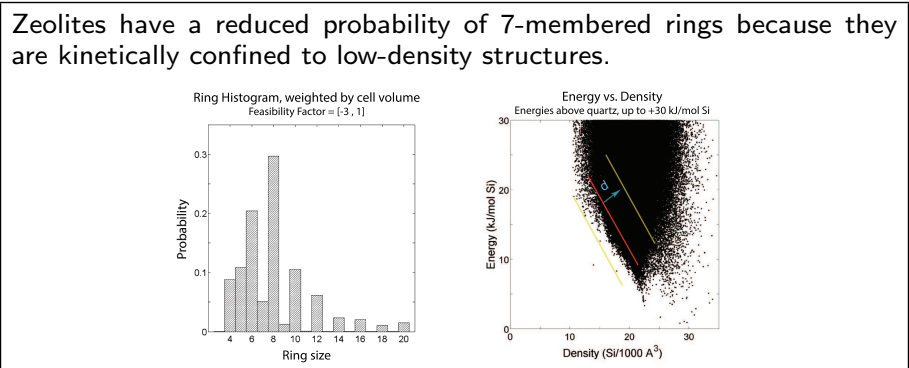

